\title{
Parallel Sequential Monte Carlo Samplers and Estimation of the Number of States in a Hidden Markov Model
}

\author{
Christopher F. H. Nam, John A. D. Aston, Adam M. Johansen \\ Department of Statistics, University of Warwick, Coventry, CV4 7AL, UK \\ \{c.f.h.nam |j.a.d.aston|a.m.johansen\}@warwick.ac.uk
}

December 3, 2012

\begin{abstract}
The majority of modelling and inference regarding Hidden Markov Models (HMMs) assumes that the number of underlying states is known a priori. However, this is often not the case and thus determining the appropriate number of underlying states for a HMM is of considerable interest. This paper proposes the use of a parallel Sequential Monte Carlo samplers framework to approximate the posterior distribution of the number of states. This requires no additional computational effort if approximating parameter posteriors conditioned on the number of states is also necessary. The proposed methodology is evaluated on a comprehensive set of simulated data and shown to compare favorably with other state of the art methods. An application to business cycle analysis is also presented.
\end{abstract}

Keywords: Hidden Markov Models; Sequential Monte Carlo; Model Selection 


\section{Introduction}

Hidden Markov Models (HMMs) provide a rich framework to model non-linear, non-stationary time series. Applications include modelling DNA sequences (Eddy, 2004), speech recognition (Rabiner, 1989) and modelling daily epileptic seizure counts for a patient (Albert, 1991).

Much of the inference and applications for HMMs such as estimating the underlying state sequence (Viterbi, 1967), parameter estimation (Baum et al., 1970) and changepoint inference (Chib (1998), Aston et al. (2011), Nam et al. (2012)), assume that the number of states the underlying Markov Chain (MC) can take, $H$, is known a priori. However, this is often not the case when presented with time series data.

Assuming a particular number of underlying states without performing any statistical analysis can sometimes be advantageous if the states correspond directly to a particular phenomena. For example in Econometric GNP analysis (Hamilton, 1989), two states are assumed a priori, "Contraction" and "Expansion", with recessions being defined as two consecutive contraction states in the underlying state sequence. Without such an assumption, this definition of a recession and the conclusions we can draw from the resulting analysis may be lost.

However, it may be necessary to assess whether such an assumption on the number of underlying states is adequate, and typically, we are presented with time series data for which we are uncertain about the appropriate number of states to assume. This paper concerns model selection for HMMs when $H$ is unknown. Throughout this paper, we use "model" and the "number of states in a HMM" interchangeably to denote the same statistical object.

Several methods for determining the number of states of a HMM currently exist. Standard model selection via maximum likelihood and Akaike's and Bayesian Information Criteria are not suitable because we can always optimise these criteria via the introduction of additional states (Titterington, 1984). In light of this, Mackay (2002) proposes an information theoretic approach which yields a consistent estimate of the number of states via a penalised minimum distance method. This frequentist approach appears to work well, although the uncertainty regarding the number of states is not explicit and relies on asymptotic arguments to obtain consistent estimates which may not be appropriate for short sequences of data.

Bayesian methods appear to dominate the model selection problem of interest, and quantify more explicitly the model uncertainty by approximating the model posterior distribution. A reversible jump Markov chain Monte Carlo (RJ-MCMC, Green (1995)) approach seems natural for such a model selection problem where the sample space varies in dimension with respect to the number of underlying states assumed and has been applied in the HMM setting by Robert et al. (2000). This is an example of VariableDimension Monte Carlo as discussed in Scott (2002). However, RJ-MCMC is often computationally intensive and care is required in designing moves such that the sampling MC mixes well both within model spaces (same number of states, different parameters) and amongst model spaces (different number of states).

Chopin and Pelgrin (2004); Chopin (2007) propose the Sequential HMM (SHMM) framework where, in addition to recording the underlying state sequence, the number of distinct states visited by the 
latent $\mathrm{MC}$ up to that time point is considered. It is this augmented MC that is considered, sampled via Sequential Monte Carlo (SMC) and used to determine the model posterior. By reformulating the problem in terms of this new augmented underlying MC and constructing the corresponding new HMM framework, they essentially turn the problem into a filtering problem and thus the use of standard particle filtering techniques are available. This setup also alleviates the problem of state identifiability as we label the states as we see them in the data sequence and is particularly suited to online applications with respect to incoming observations. This framework samples jointly the parameters and the underlying state sequence, the latter of which is particularly difficult since it is of high dimension and typically highly correlated. Unlike the other methods discussed here, the sequential nature of this approach allows its use in online applications.

Scott (2002) propose a standard Markov chain Monte Carlo (MCMC) methodology to approximate the model posterior. This uses a parallel Gibbs sampling scheme where each Gibbs sampler assumes a different number of states and is used to approximate the conditional marginal likelihood, and then combining to approximate the posterior of number of states. The use of MCMC, similarly to RJ-MCMC, requires good algorithmic design to ensure the MC is mixing well and converges.

The Bayesian methods outlined above approximate the model posterior, by jointly sampling the parameter and the state sequence, and marginalising as necessary. However, sampling the underlying state sequence can be particularly difficult, due to its high dimension and correlation, and is wasteful if the state sequence is not of interest. Alternative sampling techniques may thus be more suitable and appropriate if they can avoid having to sample the state sequence.

We take a similar approach to the parallel MCMC sampler approach of Scott (2002), in that we approximate the model posterior via the use of parallel SMC samplers, where each SMC sampler approximates the marginal likelihood and parameter posterior conditioned on the number of states. We combine these to approximate the model posterior of interest. A major advantage of the proposed approach is that the underlying state sequence is not sampled and thus less complex sampling designs can be considered. Below, we demonstrate that the SMC sampler approach can work well even with simple, generic sampling strategies. In addition, if we are already required to approximate the model parameter posteriors conditioned on several different number of states (as would be the case for sensitivity analysis, for example), the framework requires no additional computational effort and leads to parameter estimates with smaller standard errors than competing methods.

The structure of this paper is as follows: Section 2 provides a background of the statistical methods used. Section 3 outlines the proposed method. Section 4 applies the proposed methodology to both simulated data and an Econometric GNP example. Section 5 concludes the paper. 


\section{Background}

Let $y_{1}, \ldots, y_{n}$ denote a time series observed at equally spaced discrete points. One approach for modelling such a time series is via Hidden Markov Models (HMMs) which provide a sophisticated framework to model non-linear and non-stationary time series in particular. A HMM can be defined as in Cappé et al. (2005); a bivariate discrete time process $\left\{X_{t}, Y_{t}\right\}_{t \geq 0}$ where $\left\{X_{t}\right\}$ is a latent finite state Markov chain (MC), $X_{t} \in \Omega_{X}$, such that conditional on $\left\{X_{t}\right\}$, observation process $\left\{Y_{t}\right\}$ is a sequence of independent random variables where the conditional distribution of $Y_{t}$ is completely determined by $X_{t}$. We consider general finite state HMMs (including Markov switching models) such that finite dependency on previous observations and states of $X_{t}$ is permitted for an observation at time $t$. General finite state HMMs are of the form:

$$
\begin{array}{rlrl}
y_{t} \mid y_{1: t-1}, x_{1: t} & \sim f\left(y_{t} \mid x_{t-r: t}, y_{1: t-1}, \theta\right) & \text { (Emission) } \\
p\left(x_{t} \mid x_{1: t-1}, y_{1: t-1}, \theta\right) & =p\left(x_{t} \mid x_{t-1}, \theta\right) \quad t=1, \ldots, n
\end{array}
$$

Without loss of generality, we assume $\Omega_{X}=\{1, \ldots, H\}, H<\infty$, with $H$, the number of underlying states our MC can take, often being known a priori before inference is performed. $\theta$ denotes the model parameters which are unknown and consist of the transition probabilities and the state dependent emission parameters. We use the standard notation of $U_{1: n}=\left(U_{1}, \ldots, U_{n}\right)$ for any generic sequence $U_{1}, U_{2}, \ldots$

The use of HMMs allows us to compute exactly the likelihood, $l\left(y_{1: n} \mid \theta, H\right)$, via the use of the ForwardBackward algorithm (Baum et al., 1970), such that the underlying state sequence is considered exactly and does not need to be sampled. We refer the reader to MacDonald and Zucchini (1997) and Cappé et al. (2005) for good overviews of HMMs.

In dealing with unknown $\theta$, we take a Bayesian approach and consider the model parameter posterior conditioned on there being $H$ states, $p\left(\theta \mid y_{1: n}, H\right)$. This is typically a complex distribution which cannot be sampled from directly, with numerical approximations such as Monte Carlo methods being required. We turn to SMC samplers to approximate this quantity (Del Moral et al., 2006). The SMC sampler is a sampling algorithm used to sample from a sequence of distributions, $\left\{\pi_{b}\right\}_{b=1}^{B}$, defined over an arbitrary state sequence via importance sampling and resampling mechanisms. In addition, SMC samplers can be used to approximate the normalising constants, $\left\{Z_{b}\right\}_{b=1}^{B}$, for the sequence of distributions $\left\{\pi_{b}\right\}_{b=1}^{B}$ in a very natural way.

\section{Methodology}

We take a Bayesian model selection approach in determining $H$, the number of underlying states in a HMM. That is, we approximate $p\left(H \mid y_{1: n}\right)$, the posterior over the number of underlying states for a given realisation of data $y_{1: n}$ (the model posterior). Similar to the approaches of Robert et al. (2000); Scott (2002); Chopin and Pelgrin (2004); Chopin (2007), we assume a finite number of states, $H \in$ $\left\{1, \ldots, H^{\max }\right\}$. Scott $(2002)$ remark that this is a mild restriction; it is difficult to envisage using a 
model such as this without assuming $H \ll n$. Some methods, for example that of Beal et al. (2002), place no restriction on $H^{\max }$ via the use of a Dirichlet process based methodology. However, this also requires sampling the underlying state sequence via Gibbs samplers and requires approximating the likelihood via particle filters, neither of which is necessary under the proposed approach.

Via Bayes' Theorem,

$$
p\left(H \mid y_{1: n}\right) \propto p\left(y_{1: n} \mid H\right) p(H)
$$

where $p\left(y_{1: n} \mid H\right)$ denotes the marginal likelihood under model $H$, and $p(H)$ denotes the model prior. We are thus able to approximate the model posterior if we obtain the marginal likelihood associated with each model.

SMC samplers can be used to approximate the conditional parameter posterior, $p\left(\theta \mid y_{1: n}, H\right)$, and the associated marginal likelihood $p\left(y_{1: n} \mid H\right)$. We can define the sequence of distributions $\left\{\pi_{b}\right\}_{b=1}^{B}$ as follows:

$$
\pi_{b}(\theta) \propto l\left(y_{1: n} \mid \theta, H\right)^{\gamma_{b}} p(\theta \mid H), \quad b=1, \ldots, B
$$

where conditioned on a specific model $H, p(\theta \mid H)$ is the prior of the model parameters and $\gamma_{b}$ is a nondecreasing temperature schedule with $\gamma_{1}=0$ and $\gamma_{B}=1$. We thus sample initially from $\pi_{1}(\theta)=p(\theta \mid H)$ either directly or via importance sampling, and introduce the effect of the likelihood gradually. We in turn sample and approximate the target distribution, the parameter posterior $p\left(\theta \mid y_{1: n}, H\right)$. As the evaluation of the likelihood does not require sampling the underlying state sequence, the distributions defined in Equation 2 including the parameter posterior, do not require the sampling of this quantity either. Monte Carlo error is consequently only introduced through the sampling of the parameters, leading to more accurate estimates (a Rao-Blackwellised estimate). This is one of many advantages compared to other approaches such as MCMC, where the underlying state sequence needs to be sampled.

Note that this setup is different to that proposed in Chopin and Pelgrin (2004) and Chopin (2007), where distributions are defined as $\pi_{b}=p\left(\theta, x_{1: b} \mid y_{1: b}\right)$ with respect to incoming observations. In addition to the proposed approach not simulating $x_{1: b}$, a different tempering schedule is employed due to the increasing data sequence over time. The data tempering approach of Chopin (2007) facilitates online estimation; such a schedule could also be employed here but the nature of the computations involved are such that it would not lead to such substantial efficiency gains in our setting and we have preferred the geometric tempering approach which leads to a more regular sequence of distributions.

$Z_{B}$, the normalising constant for the parameter posterior $p\left(\theta \mid y_{1: n}, H\right)=p\left(\theta, y_{1: n} \mid H\right) / Z_{B}$, is more specifically of the following form,

$$
Z_{B}=\int l\left(y_{1: n} \mid \theta, H\right) p(\theta \mid H) d \theta=\int p\left(y_{1: n}, \theta \mid H\right) d \theta=p\left(y_{1: n} \mid H\right) .
$$

That is, the normalising constant for the parameter posterior conditioned on model $H$, is the conditional marginal likelihood of interest. Given that we can approximate the marginal likelihood, we can thus approximate the model posterior as follows: 


\section{Algorithm outline:}

1. For $h=1, \ldots, H^{\max }$,

(a) Approximate $p\left(y_{1: n} \mid H=h\right)$ and $p\left(\theta \mid y_{1: n}, H=h\right)$, the marginal likelihood (see Section 3.1) and parameter posterior (see Nam et al. (2012)) conditioned on $h$ states, via SMC samplers.

2. Approximate $p\left(H=h \mid y_{1: n}\right)$, the model posterior, via the approximation of $p\left(y_{1: n} \mid H=h\right)$ and model prior $p(H)$.

\subsection{Approximating $p\left(y_{1: n} \mid H\right)$}

SMC samplers can also be used to approximate normalising constants, $Z_{b}$, for the sequence of distributions, $\pi_{b}, b=1, \ldots, B$. SMC samplers work on the principle of providing weighted particle approximations of distributions through importance sampling and resampling techniques. For a comprehensive exposition of SMC samplers, we refer the reader to Del Moral et al. (2006).

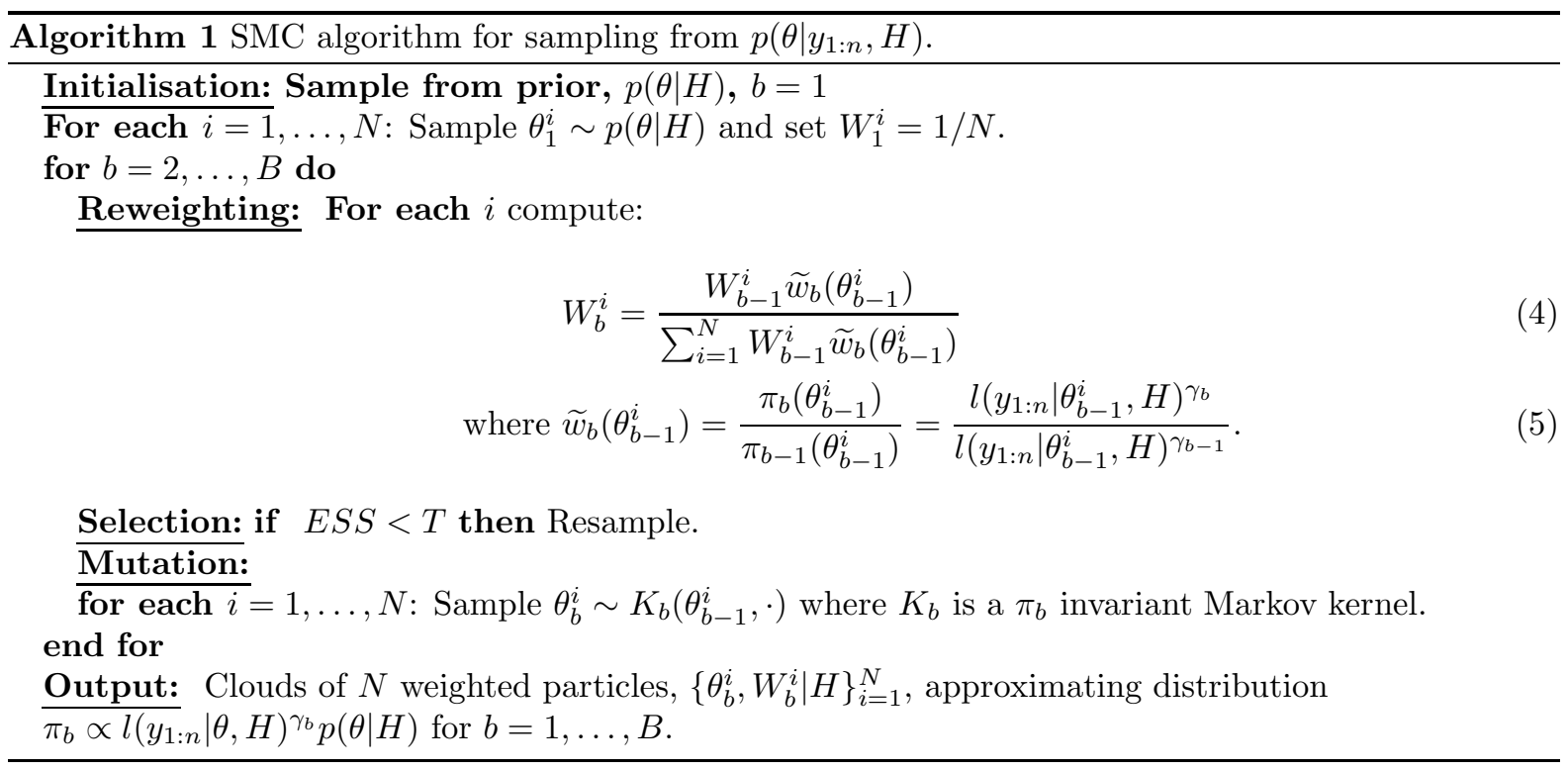

Using the formulation of SMC samplers within HMMs from Nam et al. (2012), reproduced here as Algorithm 1, the main output of the SMC samplers algorithm is a series of weighted sample approximations of $\pi_{b}$, namely $\left\{\theta_{b}^{i}, W_{b}^{i} \mid H\right\}_{i=1}^{N}$, where $N$ is the number of samples used in the SMC approximation. The approximation of the ratio between consecutive normalising constants can then be found as:

$$
\frac{Z_{b}}{Z_{b-1}} \approx \widehat{\frac{Z_{b}}{Z_{b-1}}}=\sum_{i=1}^{N} W_{b-1}^{i} \widetilde{w}_{b}\left(\theta_{b-1}^{i}\right):=\bar{W}_{b} .
$$

This ratio corresponds to the normalising constant for weights at iteration $b . Z_{B}$, can thus be approximated as:

$$
\widehat{Z_{B}}=\widehat{Z}_{1} \prod_{b=2}^{B} \bar{W}_{b}
$$


which, remarkably, is an unbiased estimator of the true normalising constant (Del Moral, 2004).

Note that the normalising constant, $Z_{b}$, corresponds to the the following quantity

$$
\pi_{b}(\theta)=\frac{\varphi_{b}(\theta)}{Z_{b}}
$$

where $\varphi_{b}$ is the unnormalised density. We can thus approximate the marginal likelihood by simply recording the normalising constants for the weights, $\bar{W}_{b}$, at each iteration of Algorithm 1.

There is a great deal of flexibility with the SMC implementation and some design decisions are necessarily dependent upon the model considered. We have found that a reasonably straightforward strategy works well for the class of HMMs which we consider.An example implementation, similar to that discussed in Nam et al. (2012), is as follows: we set $\gamma_{b}=\frac{b-1}{B-1}$. As transition probabilities matrices are a fundamental component in HMMs, we initialise as follows: consider the transition probability vectors, $p_{h}=\left(p_{h 1}, \ldots, p_{h H}\right), h=1, \ldots, H$ such that $\mathbf{P}=\left\{p_{1}, \ldots, p_{H}\right\}$, and sample from the prior $p_{h} \stackrel{\text { iid }}{\sim} \operatorname{Dir}\left(\alpha_{h}\right), h=1, \ldots, H$ where $\alpha_{h}$ is a $H$-long hyperparameter vector. As HMMs are associated with persistent behaviour, we choose $\alpha_{h}$ which reflects this type of behaviour. Relatively flat priors are generally implemented for the emission parameters. Random Walk Metropolis Hastings proposal kernels can be used for the mutation step of the algorithm. Details of specific implementation choices are given for representative examples in the following section.

\section{Results}

This section applies the proposed methodology to a variety of simulated and real data. All results have been obtained using the approach of Section 3 with the following settings. $N=500$ samples and $B=100$ iterations have been used to approximate the sequence of distributions. Additional sensitivity analysis has been performed with respect to larger values of $N$ and $B$ which we found reduced the Monte Carlo variability of estimates, as would be expected, but for practical purposes samples of size 500 were sufficient to obtain good results. $\alpha_{h}$ is a $H$-long hyperparameter vector full of ones, except in the $h$-th position where a 10 is present. This encourages the aforementioned persistent behaviour in the underlying MC associated with HMMs. The linear tempering schedule and proposal variances used have not been optimised to ensure optimal acceptance rates. Promising results are obtained with these simple default settings.

For the model selection results, a uniform prior has been assumed over the model space in approximating the model posterior. We consider selecting the maximum a posterior (MAP) model, that is $\arg \max _{h=1, \ldots, H^{\max }} p\left(H=h \mid y_{1: n}\right)$, as this indicates the strongest evidence for the model favoured by the observed data.

\subsection{Simulated Data}

We consider simulated data generated by two different models; Gaussian Markov Mixture (GMM) and Hamilton's Markov Switching Autoregressive model of order $r$ (HMS-AR(r), Hamilton (1989)). The first 
model has been chosen due to its relative simplicity and connection to mixture distributions, and the latter is a more sophisticated model which can be used to model Econometric GNP data (Hamilton, 1989) and brain imaging signals (Peng et al., 2011). HMS-AR models can be seen as an extension of GMM models such that only the underlying mean switches, the variance is state invariant, and dependency on previous observations is induced in an autoregressive nature into the mean. For various scenarios under these two models, we present an example realisation of the data from the same seed (left column) and the model selection results from 50 simulations (right column). Changes in state in the underlying state sequence occur at times 151, 301 and 451 . We consider a maximum of five states, $H^{\max }=5$, as we believe that no more than five states are required to model the business cycle data example we will consider later, and the simulations are designed to reflect this.

The following priors have been used for the state dependent mean and precision (inverse of variance) parameters: $\mu_{h} \stackrel{\text { iid }}{\sim} \mathrm{N}(0,100), \frac{1}{\sigma_{h}^{2}} \stackrel{\text { iid }}{\sim}$ Gamma(shape $=1$, scale $\left.=1\right), h=1, \ldots, H$. For the HMS-AR model, we consider the partial autocorrelation coefficients (PAC, $\left.\psi_{1}\right)$ in place of AR parameter, $\phi_{1}$, with the following prior, $\psi_{1} \sim \operatorname{Unif}(-1,1)$. The use of PAC allows us to maintain stationarity amongst the AR coefficients more efficiently. Baseline proposal variances of 10 have been used for each parameters' mutation step which decrease linearly as a function of sampler iteration. For example, the proposal variance $\frac{\sigma_{\mu}^{2}}{b}$ is used for $\mu_{h}$ mutations.

\subsubsection{Gaussian Markov Mixture}

Figure 1 displays a variety of results generated by a GMM model under the proposed parallel SMC methodology. In addition, we compare our model selection results to the Sequential Hidden Markov Model (SHMM) approach as proposed in Chopin (2007) where computer code for a GMM is freely available (http://www.blackwellpublishing.com/rss/Volumes/Bv69p2.htm). The following settings have been used for the SHMM implementation; $N=5000$ samples have been used to approximate the sequence of distributions, $\pi_{b}^{\prime}=p\left(\theta, x_{1: b} \mid y_{1: b}\right), H^{\max }=5$ as the maximum number of states possible and one SMC replicate per dataset. The same prior settings under the proposed parallel SMC samplers have been implemented. Other default settings in the SHMM code such as model averaging being performed have been utilised. The model posterior approximations from this approach are displayed alongside the parallel SMC posterior approximations.

Figures 1(a) and 1(b) concern a simple two state scenario with changing mean and variance simultaneously. From the data realisation, it is evident that two or more states are appropriate in modelling such a time series. This is reflected in the model selection results with a two state model being significantly the most probable under the model posterior from all simulations, and always correctly selected under MAP. However, uncertainty in the number of appropriate states is reflected with probability assigned to a three state model amongst the simulations. These results indicate that the proposed methodology works well on a simple, well defined toy example. Results concur with the SHMM framework; a two state model is most probable for all simulations, and less model uncertainty is exhibited. 
Figure 1(c) and 1(d) displays results from a similar three state model, where different means correspond to the different states with subtle changes in mean present, for example around the 151 time point. Such subtle changes are of interest. The correct number of states is significantly the most probable under all simulations, and always correctly identified under MAP selection. Under the SHMM approach, more variability is present amongst the simulations. A three state model is largely the most probable, although some approximations display a four or two state model also being the most probable.

Figure 1(e) and 1(f) displays results from a challenging scenario of changes in both subtle mean and variance, independently of each other, with four states being present. The SMC methodology is unable to correctly identify the number of states, with three states being the most probable and most selected model from the majority of the simulations. However, given the example data realisation, it is not particularly surprising that a three state model has been selected; it is not entirely evident that the realisation is generated from a four or even three state model. This underfitting in the number of states is suspected to have occurred due to the segment of data from 451 to 512 being too short and associated with the previous segment of data from the previous state. Some probability has been associated with four and two state models however. In addition the variability in the approximation of the model posterior is more pronounced for this simulation scenario, a result of the challenging scenario presented. The SHMM also performs similarly, with probability being assigned to two state and three state models.

Figure 1(g) and 1(h) presents results from a one state GMM model, a stationary Gaussian process. Of interest here is whether our methodology is able to avoid overfitting even though a true HMM is not present. The model selection results highlight that overfitting is successfully avoided with a one state model being most probable under the model posterior for all simulations and always the most selected under MAP. The SHMM method, however, attaches substantial probability to a two state model over a one state model.

We also consider comparing the samples approximating the true emission parameters under the two methods. We consider the presented data scenarios of Figures 1(a) and 1(c) where the proposed SMC and SHMM method both concur with respect to the number of underlying states identified via MAP. Table 1 displays the averaged posterior means and standard error for each emission parameter over the 50 simulations. The SMC methodology is more accurate in estimating the true value, and the standard error is smaller compared to the estimates provided by SHMM. This is as expected since the SHMM methodology requires sampling the underlying state sequence which induces the sampling error in the standard error of the estimates. This does not occur under the parallel SMC approach.

Figure 2 displays box plots of the posterior means (2(a) and 2(c)) and standard error (2(b) and $2(d))$ of the emission parameter estimates for all 50 simulations. The posterior mean box plots indicate further that the proposed parallel SMC approach is generally more accurate and centered around the true emission parameter values (horizontal red dotted lines) for all simulations. The SHMM estimates are generally less precise with a greater range of values present. Similarly, the standard error box plots 


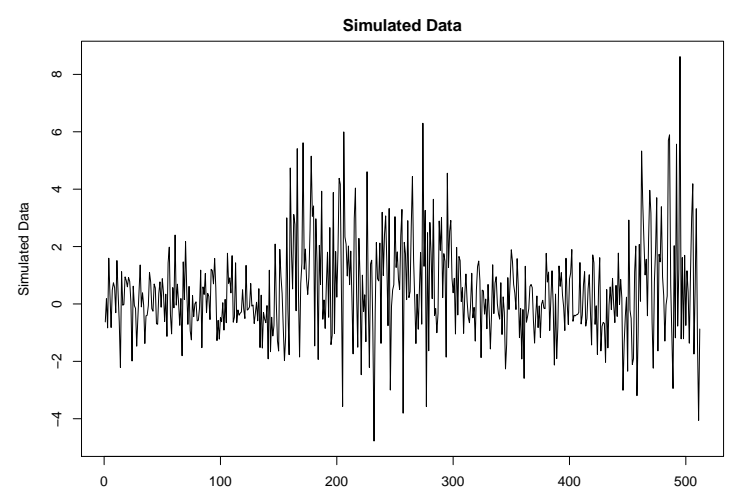

(a) GMM Data, 2 states $\left(\left\{\mu_{1}=0, \sigma_{1}^{2}=1\right\},\left\{\mu_{2}=1, \sigma_{2}^{2}=\right.\right.$ 4\})

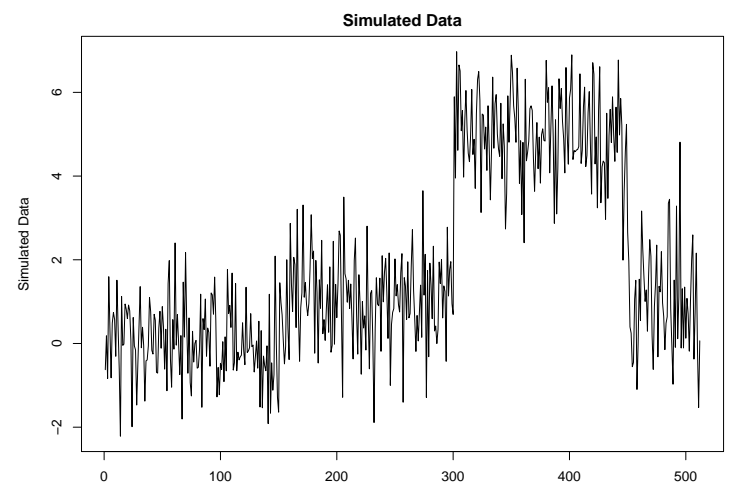

(c) GMM Data, 3 states, $\left(\left\{\mu_{1}=0, \sigma_{1}^{2}=1\right\},\left\{\mu_{2}=1, \sigma_{2}^{2}=\right.\right.$ $\left.1\},\left\{\mu_{3}=5, \sigma_{3}^{2}=1\right\}\right)$

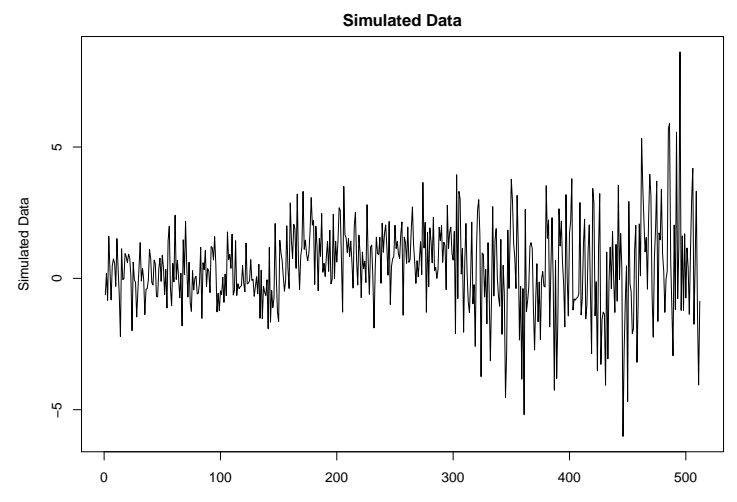

(e) GMM Data, 4 states, $\left(\left\{\mu_{1}=0, \sigma_{1}^{2}=1\right\},\left\{\mu_{2}=1, \sigma_{3}^{2}=\right.\right.$ $\left.1\},\left\{\mu_{3}=0, \sigma_{3}^{2}=4\right\},\left\{\mu_{4}=1, \sigma_{4}^{2}=4\right\}\right)$

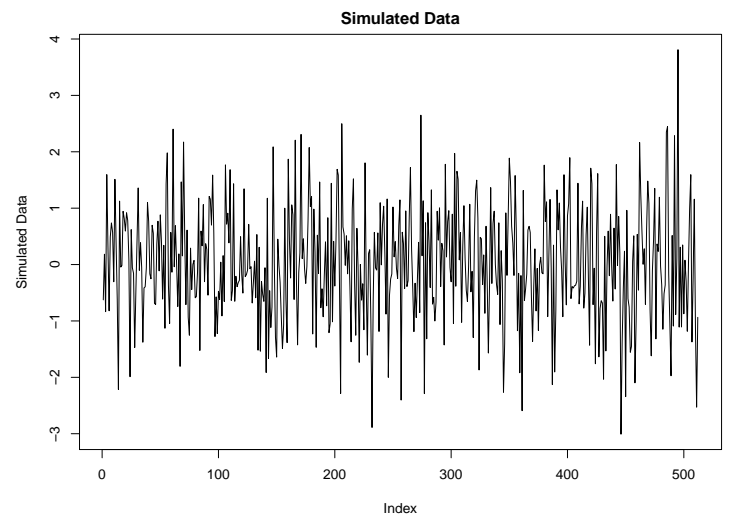

(g) GMM Data, 1 state, $\left(\left\{\mu_{1}=0, \sigma_{1}^{2}=1\right\}\right)$

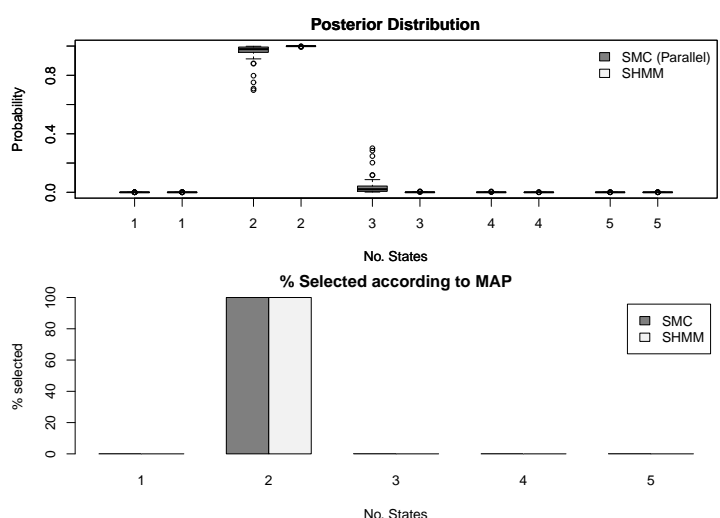

(b) GMM Model Selection Results, 2 states

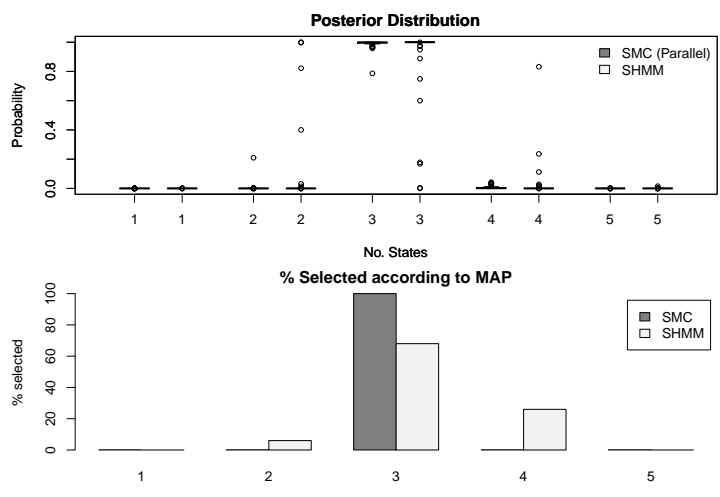

(d) GMM Model Selection Results, 3 states

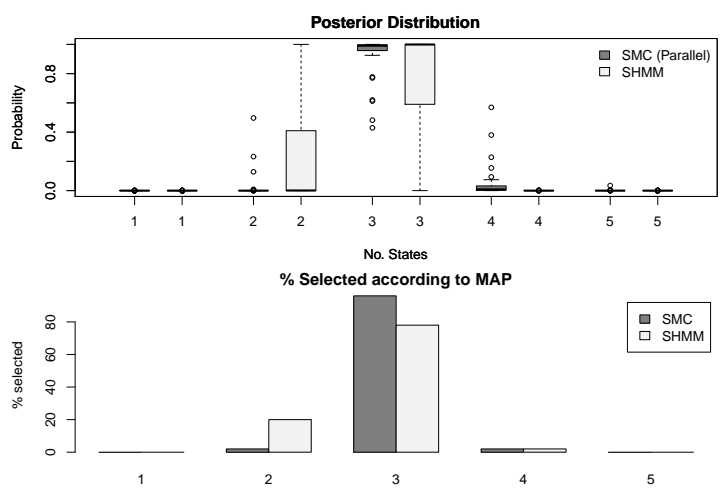

(f) GMM Model Selection Results, 4 states

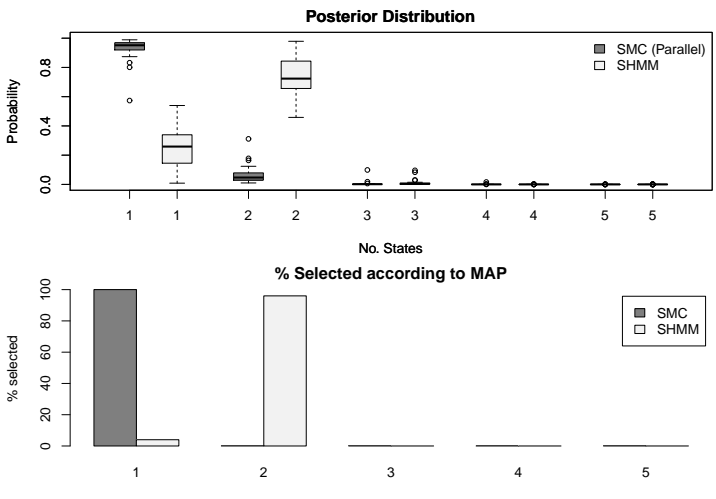

(h) GMM Model Selection Results, 1 state

Figure 1: Model Selection Results for variety of Gaussian Markov Mixture Data. Left column shows examples of data realisations, right column shows the model selection results; boxplots of the model posterior approximations under the parallel SMC and SHMM approaches, and percentage selected according to maximum a posterior (MAP). Results are from 50 realisations. 


\begin{tabular}{|r||r|r|r||r|r|r|}
\hline \hline & $\mu_{1}$ & $\mu_{2}$ & $\mu_{3}$ & $\sigma_{1}$ & $\sigma_{2}$ & $\sigma_{3}$ \\
\hline \hline Truth & 0 & 1 & - & 1 & 2 & - \\
\hline SMC & $0.00(0.06)$ & $0.99(0.14)$ & - & $1.00(0.04)$ & $2.03(0.10)$ & - \\
\hline SHMM & $0.05(0.18)$ & $0.94(0.23)$ & - & $1.06(0.19)$ & $1.97(0.21)$ & - \\
\hline \hline Truth & 0 & 1 & 5 & 1 & 1 & 1 \\
\hline SMC & $0.00(0.09)$ & $1.00(0.08)$ & $5.00(0.08)$ & $1.00(0.06)$ & $1.01(0.05)$ & $1.01(0.06)$ \\
\hline SHMM & $0.70(0.19)$ & $1.50(0.38)$ & $3.51(33.86)$ & $1.01(0.06)$ & $1.01(0.06)$ & $1.07(0.35)$ \\
\hline \hline
\end{tabular}

Table 1: Averaged posterior means and standard error for each emission parameter over the 50 simulations for the two data scenarios considered. We compare the proposed parallel SMC and SHMM method. Averaged standard errors are denoted in the parentheses. Results indicate that the SMC outperforms the SHMM method with greater accuracy in approximating the true values and smaller standard errors.

indicate that the standard error is less for the proposed SMC methodology compared to the SHMM method, presumably due to the lower dimension of the sampling space.

The results indicates that in addition to identifying the correct model more often, more accurate estimates are obtained under the proposed SMC approach, compared to the existing SHMM method. This is a result of the Rao-Blackwellised estimator provided by the SMC samplers framework and despite more samples being used under the SHMM approach. As fewer samples are required to achieve good, accurate estimates, the proposed parallel SMC method would appear to be more computationally efficient. In addition, while not directly comparable, the runtime for the SMC samplers approach for one time series was approximately 15 minutes to consider the five possible model orders using $N=500$ particles (implemented in R (R Development Core Team, 2011)), while it was approximately 90 minutes for the SHMM approach with the default $N=5000$ particles (implemented in MATLAB (MATLAB, 2012)). 


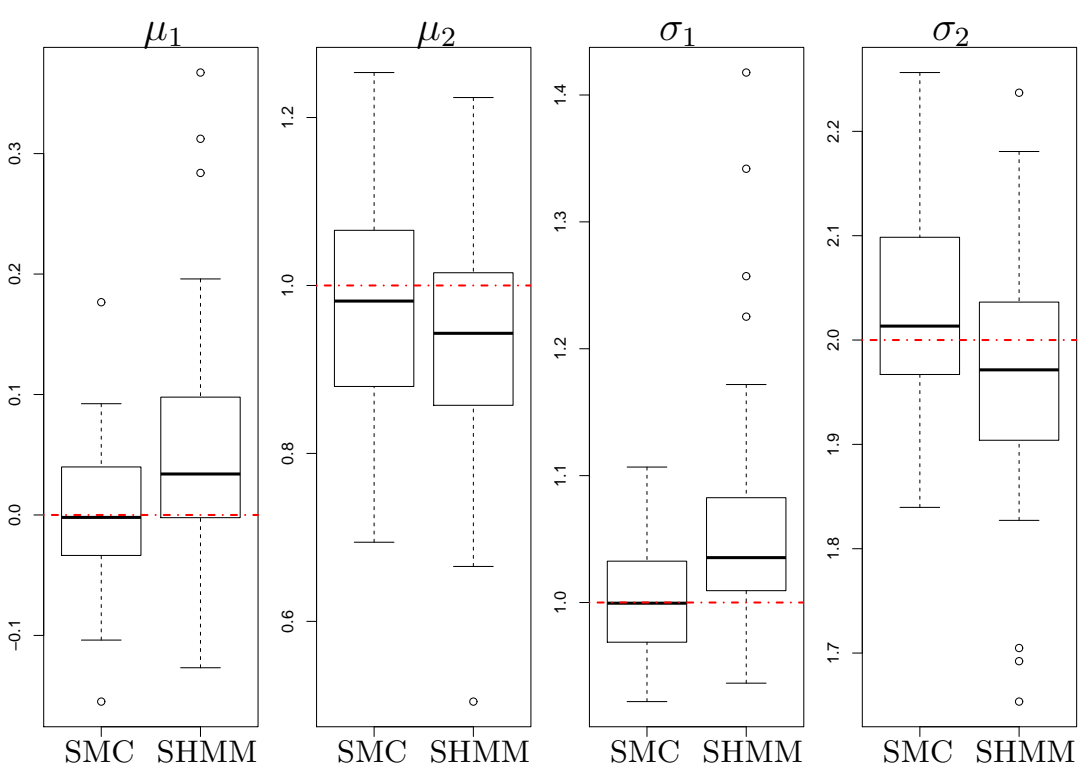

(a) Posterior mean of emission parameters, 2 states

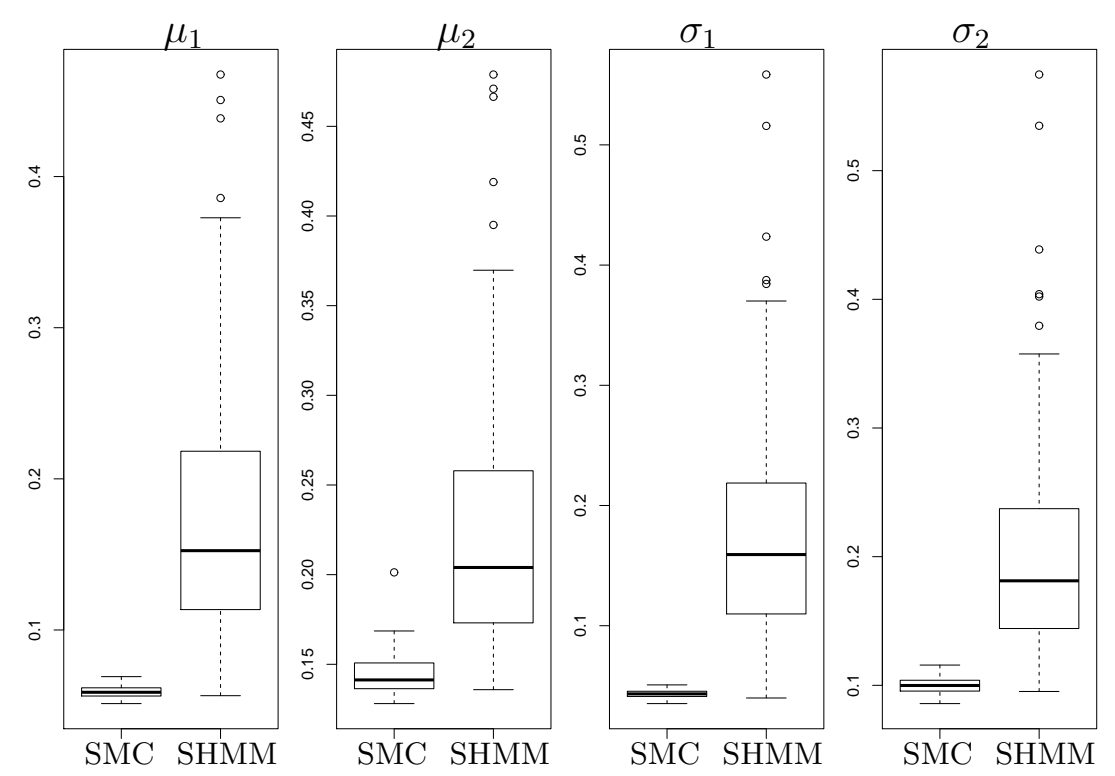

(b) Posterior standard error of emission parameters, 2 states
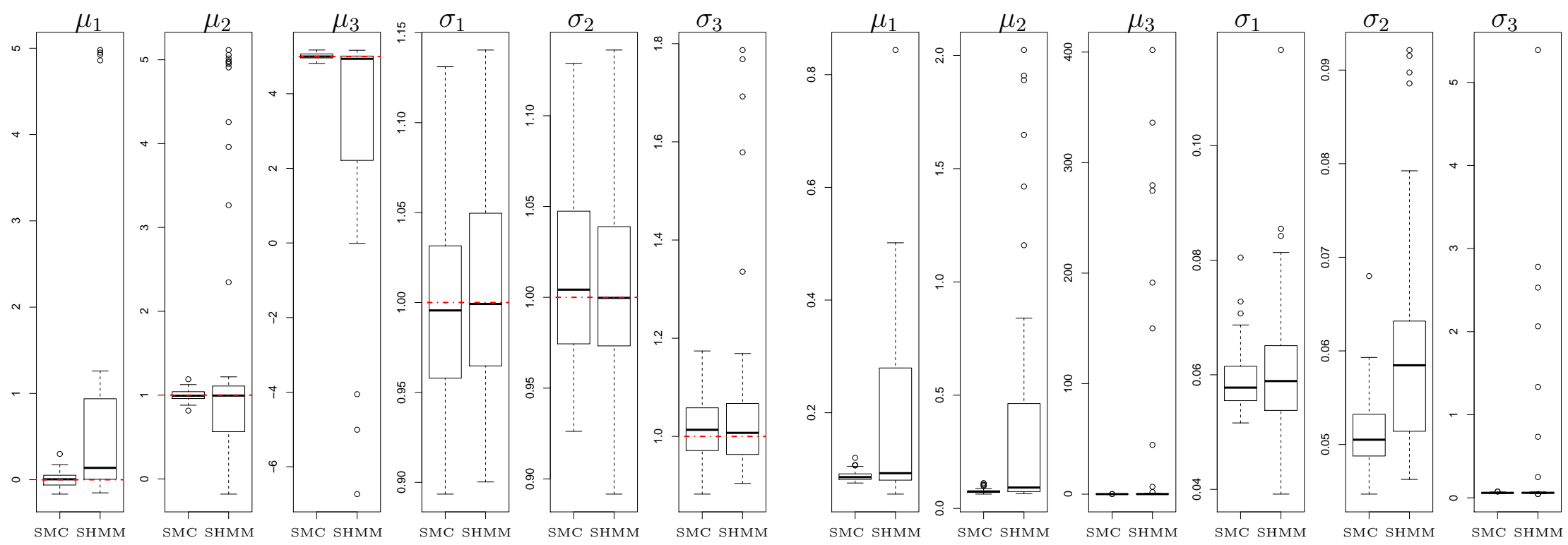

(c) Posterior mean of emission parameters, 3 states

(d) Posterior standard error of emission parameters, 3 states

Figure 2: Box plots of the posterior means and standard error for each emission parameter over 50 simulations. Red dotted values denote the value of the emission parameter used to generate the simulated data in the posterior mean box plots. We compare the results under the two approaches, parallel SMC and SHMM. We observe that the proposed SMC approach fares better with posterior means centered more accurately around the true values, and the standard error for the samples being smaller. 


\subsubsection{Hamilton's Markov Switching Autoregressive Model of order $r$, HMS-AR( $(r)$}

Figure 3 shows results from a HMS-AR model with autoregressive order one; we assume that this autoregressive order is known a priori although the proposed methodology could easily be extended to consider model selection with respect to higher AR orders. The following results were obtained using data generated using a two state model, with varying autoregressive parameter, $\phi_{1}$, and the same means and variance used for each scenario $\left(\mu_{1}=0, \mu_{2}=2, \sigma^{2}=1\right)$. Interest lies in how sensitive the model selection results are with respect to $\phi_{1}$. For small values of $\phi_{1}$ (for example $\phi_{1}=0.1,0.5$ ) indicating small dependency on previous observations, our proposed methodology works well with the correct number of true states being highly probable and always the most selected according to MAP. Relatively little variability exists in the approximation of the model posterior. However, as $\phi_{1}$ begins to increase and tend towards the unit root, for example $\phi_{1}=0.9$, we observe that more uncertainty is introduced into the model selection results, with greater variability in the model posterior approximations and alternative models being selected according to MAP. However, as the data realisation in Figure 3(g) suggests, the original two state model is hard to identify by eye and thus our methodology simply reflects the associated model uncertainty. These results indicate that the proposed model selection method works for sophisticated models such as HMS-AR models, although the magnitude of the autoregressive nature may affect results.

\subsection{Hamilton's GNP data}

Hamilton's GNP data (Hamilton, 1989) consists of differenced quarterly logarithmic US GNP between the time periods 1951:II to 1984:IV. Hamilton (1989) and Aston et al. (2011) model $y_{t}$, the aforementioned transformed data, by a two state HMS-AR(4) model, before performing analysis regarding identification of starts and ends of recessions. The two underlying states denote "Contraction" and "Expansion" states in order to correspond directly with the definition of a recession; two consecutive quarters of contraction. Whilst such a model works in practice for recession inference, we investigate whether a two state HMS$\mathrm{AR}(4)$ model is indeed appropriate. We assume the autoregressive order of four, is known a priori relating to annual seasonality, and is adequate in modelling the data. We assume a maximum of five possible states in the HMM framework $\left(H^{\max }=5\right)$ as we believe that the data arises from at most five possible states for the particular time period considered.

The following priors have been used: for the means, $\mu_{h} \stackrel{\text { iid }}{\sim} \mathrm{N}(0,10), h=1, \ldots, H$, precision (inverse variance) $\frac{1}{\sigma^{2}} \sim$ Gamma(shape $=1$, scale $=1$ ), PAC coefficients $\psi_{j} \stackrel{\text { iid }}{\sim} \operatorname{Unif}(-1,1), j=1, \ldots, 4 . \quad$ A uniform prior has been used over the number of states $H$. The baseline proposal variance is 10 which diminishes linearly with each iteration of the sampler.

Figure 4 displays the corresponding dataset and model selection results from 10 different SMC replicates. The model selection results, Figure 4(b), demonstrate that there is uncertainty in the appropriate number of underlying states with non-negligible probability assigned to each model considered and variability amongst the SMC replication results. Some of the alternative models seem plausible, for example 


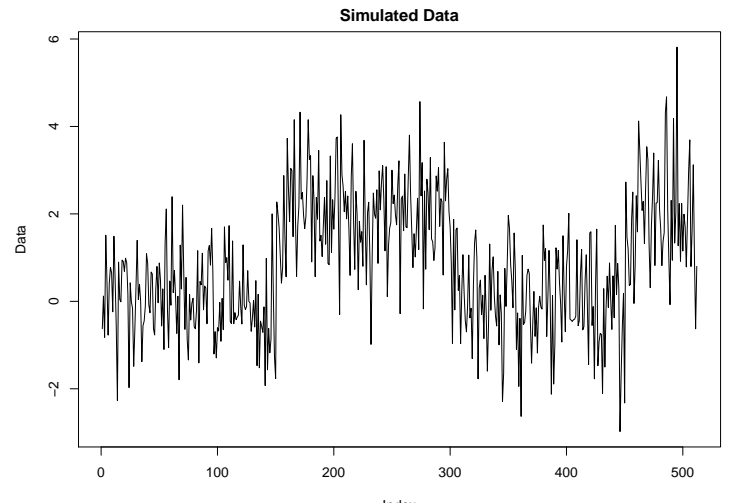

(a) HMS-AR Data, 2 indax states, $\phi_{1}=0.1$

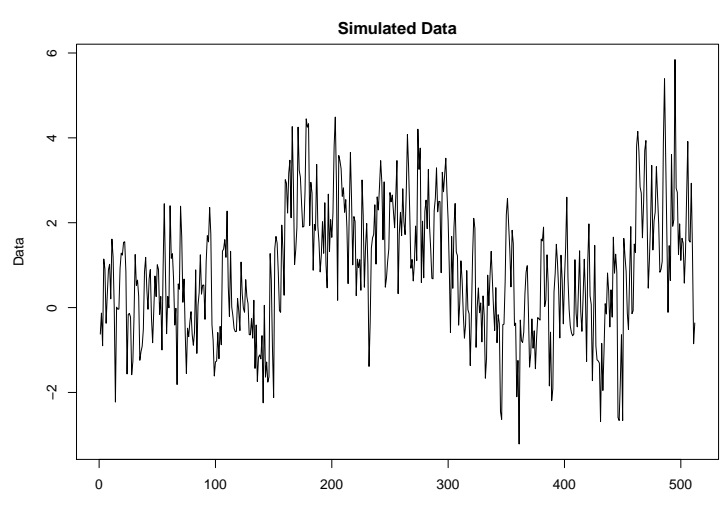

(c) HMS-AR Data, 2 states, $\phi_{1}=0.5$

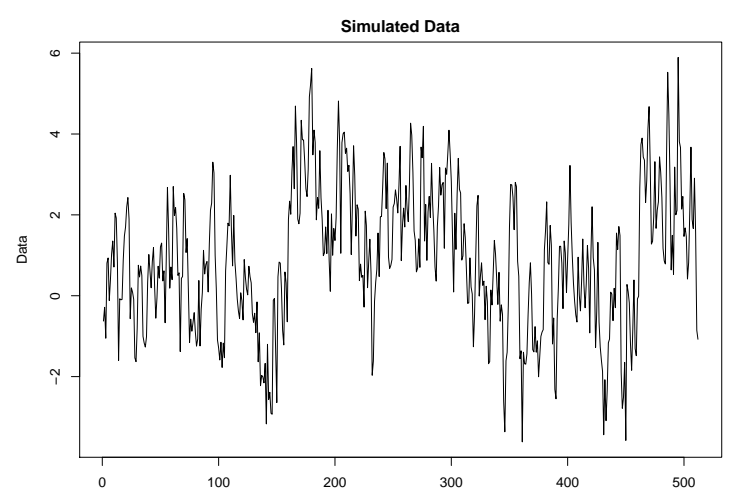

(e) HMS-AR Data, 2 states, $\phi_{1}=0.75$

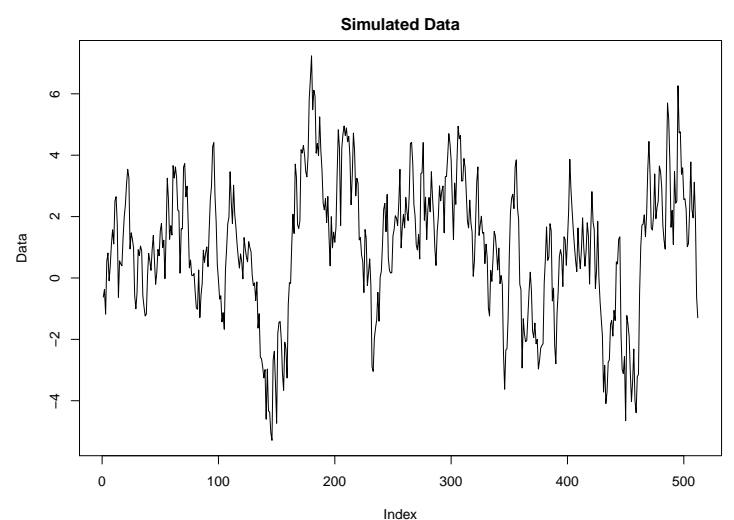

(g) HMS-AR Data, 2 states, $\phi_{1}=0.9$
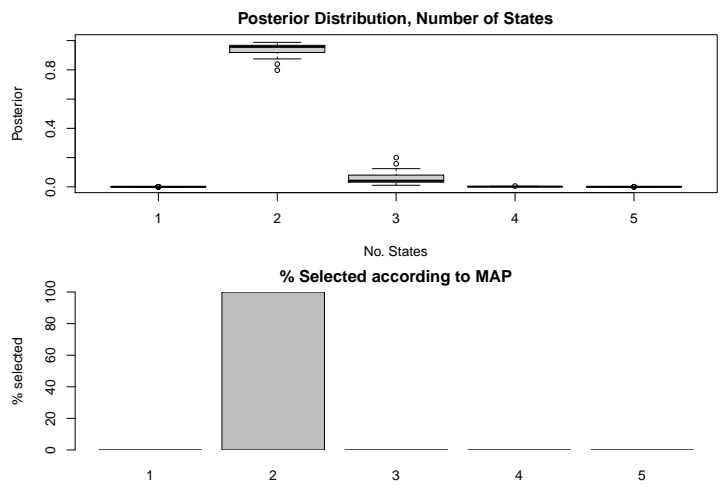

(b) HMS-AR Model Selection Results, 2 states, $\phi_{1}=0.1$
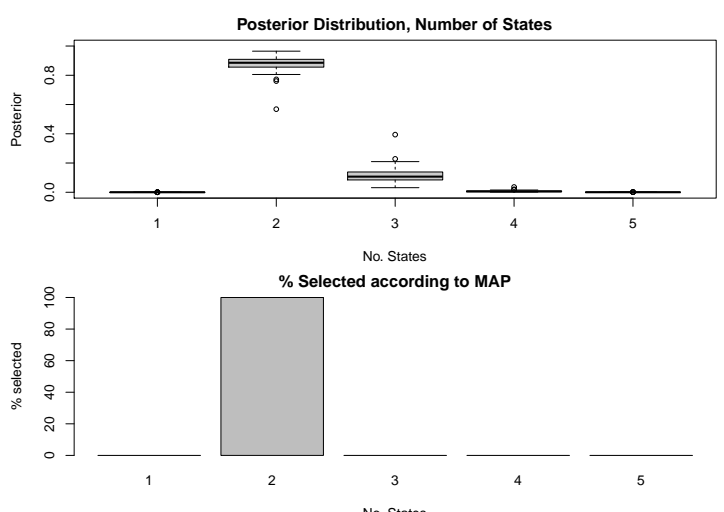

(d) HMS-AR Model Selection Results, 2 states, $\phi_{1}=0.5$

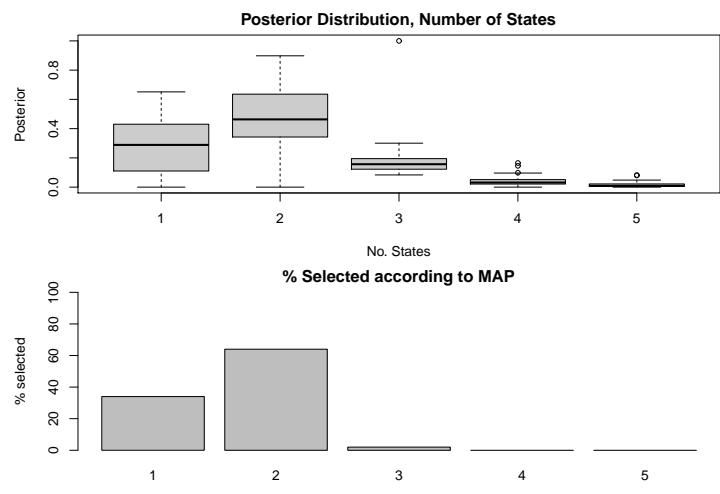

(f) HMS-AR Model Selection Results, 2 states, $\phi_{1}=0.75$

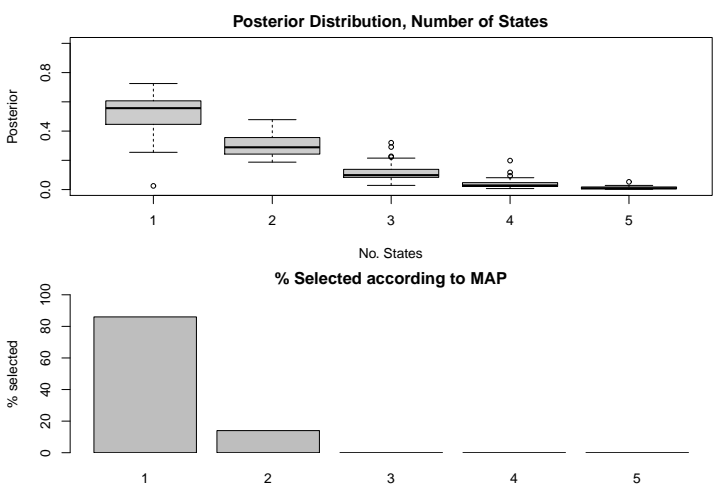

(h) HMS-AR Model Selection Results, 2 states, $\phi_{1}=0.9$

Figure 3: Model Selection Results for variety of HMS-AR(1) data with $\mu_{1}=0, \mu_{2}=2, \sigma^{2}=1$ and varying $\phi_{1}$. Left column shows examples of data realisations, right column shows the parallel SMC model selection results from 50 realisations; approximations of the model posterior, and percentage selected according to maximum a posterior (MAP). 
a one-state model given the plot of the data and the additional underlying states modelling the subtle nuances and features in the data. However, a two state model is the most selected the most according to MAP. In addition, the distribution appears to tail off as we consider more states, thus indicating that value of $H^{\max }$ used is appropriate. In conclusion, the two state HMS-AR(4) model assumed by Hamilton (1989) does seem adequate in modelling the data although this is not immediately evident and uncertainty is associated with the number of underlying states.

Note that the variability in the model selected using MAP between repeated runs is due to the small but non-negligible sampling variability in the posterior model probabilities. Additional simulations (not shown) verify that moderate increases in the SMC sample size, $N$, are sufficient to eliminate this variability.

\section{Conclusion and Discussion}

This paper has proposed a methodology in which the number of underlying states in a HMM framework, $H$, can be determined by the use of parallel Sequential Monte Carlo samplers. Conditioned on the number of states, the conditional marginal likelihood can be approximated in addition to the parameter posterior via SMC samplers. By conditioning of a different number of states, we can obtain several conditional marginal likelihoods. These conditional marginal likelihoods can then be combined with an appropriate prior to approximate the model posterior, $p\left(H \mid y_{1: n}\right)$, of interest. The use of SMC samplers within a HMM framework results in an computationally efficient and flexible framework such that the underlying state sequence does not need to be sampled unnecessarily compared to other methods which reduces Monte Carlo error of parameter estimates, and complex design algorithms are not required.

The proposed methodology has been demonstrated on a variety of simulated data and GNP data and shows good results, even in challenging scenarios where subtle changes in emission parameters are present. The results on the GNP data have further confirmed that a two state HMS-AR model assumed in previous studies and analysis is appropriate, although the uncertainty associated with the number of underlying states has now been captured. In the settings considered, the method performs at least as well as other state of the art approaches in the literature such as the SHMM approach proposed in Chopin (2007).

From a modelling perspective, the model selection results presented in this paper have assumed a uniform prior over the collection models considered but there would be no difficulty associated with the use of more complex priors. Perhaps more important in the context of model selection is the specification of appropriate priors over model parameters, which can have a significant influence on model selection results: some sensitivity analysis should always be conducted to assess the impact of parameter priors on model selection results obtained by any method. From the perspective of computational efficiency it is desirable to identify a value of $H^{\text {max }}$ which is sufficiently large to allow for good modelling of the data but not so large that the computational cost of evaluating all possible models becomes unmanageable 


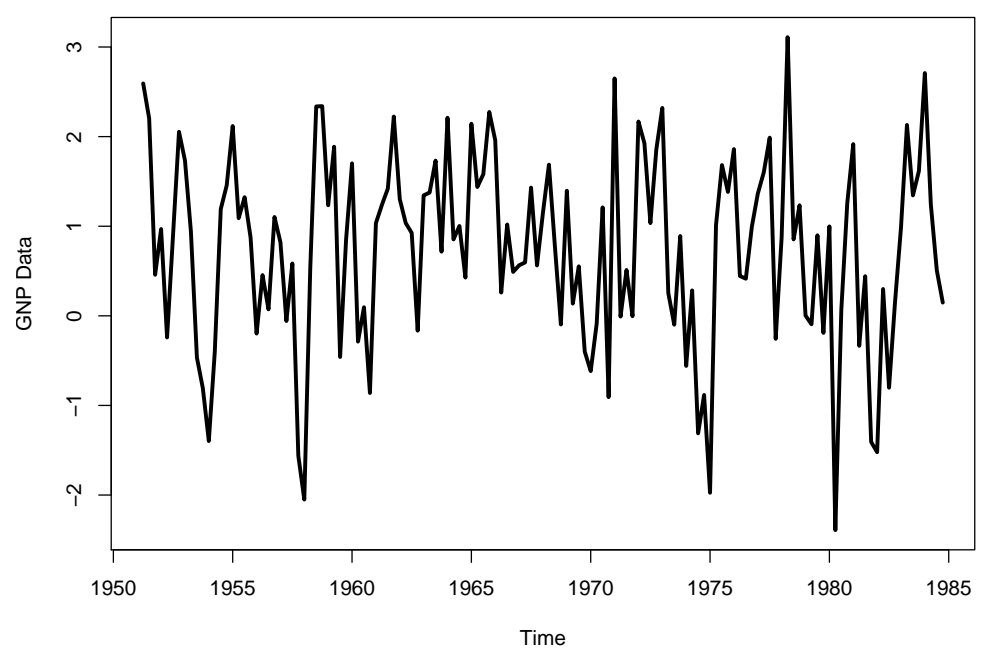

(a) Hamilton's GNP data: differenced quarterly logarithmic US GNP between 1951:II to 1984:IV.
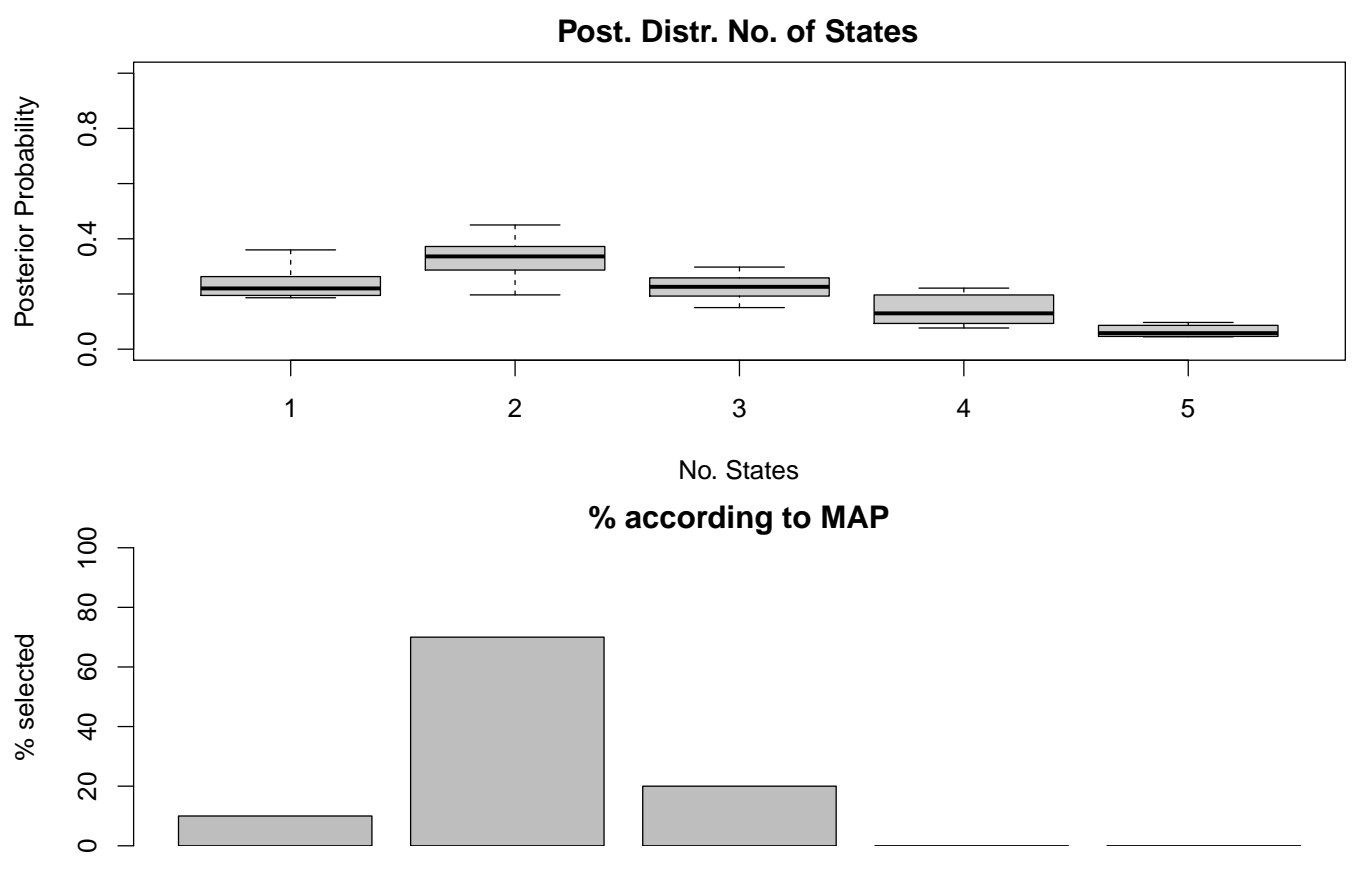

$\%$ according to MAP

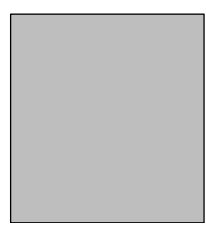

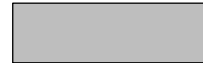

No. States

(b) Model Selection Results for GNP data

Figure 4: Model selection results for Hamilton's GNP data under the proposed model selection methodology. 4(a) displays the analysed transformed GNP data. 4(b) displays the model posterior approximations from 10 SMC replications, and percentage selected under maximum a posterior (MAP) 
(noting that the cost of dealing with any given model is, with the proposed method as well as most others, an increasing function of the complexity of that model).

Finally, we note that, although this paper has focused predominantly on a retrospective, offline context, in an online context it would be possible to consider the sequence of distributions defined by $\pi_{b}^{\prime}(\theta) \propto l\left(y_{1: b} \mid \theta, H\right) p(\theta \mid H)$, rather than $\pi_{b}(\theta) \propto l\left(y_{1: n} \mid \theta, H\right)^{\gamma_{b}} p(\theta \mid H)$ in a similar spirit to the SHMM approach but without the simulation of the latent state sequence.

\section{Acknowledgements}

The authors would like to thank Nicolas Chopin for making available the computer code for the SHMM algorithm.

\section{References}

Albert, P. S. (1991). A two-state Markov mixture model for a time series of epileptic seizure counts. Biometrics 47(4), 1371-1381.

Aston, J. A. D., J. Y. Peng, and D. E. K. Martin (2011). Implied distributions in multiple change point problems. Statistics and Computing 22, 981-993.

Baum, L. E., T. Petrie, G. Soules, and N. Weiss (1970). A maximization technique occurring in the statistical analysis of probabilistic functions of Markov chains. The Annals of Mathematical Statistics 41(1), pp. $164-171$.

Beal, M., Z. Ghahramani, and C. Rasmussen (2002). The infinite hidden Markov model. Advances in neural information processing systems 14, 577-584.

Cappé, O., E. Moulines, and T. Rydén (2005). Inference in Hidden Markov Models. Springer Series in Statistics.

Chib, S. (1998). Estimation and comparison of multiple change-point models. Journal of Econometrics $86,221-241$.

Chopin, N. (2007). Inference and model choice for sequentially ordered hidden Markov models. Journal of the Royal Statistical Society Series B 69(2), 269.

Chopin, N. and F. Pelgrin (2004). Bayesian inference and state number determination for hidden Markov models: an application to the information content of the yield curve about inflation. Journal of Econometrics 123(2), 327 - 344. Recent advances in Bayesian econometrics.

Del Moral, P. (2004). Feynman-Kac formulae: genealogical and interacting particle systems with applications. Probability and Its Applications. New York: Springer.

Del Moral, P., A. Doucet, and A. Jasra (2006). Sequential Monte Carlo samplers. Journal of the Royal Statistical Society Series B 68(3), 411-436.

Eddy, S. R. (2004). What is a hidden Markov model? Nature Biotechnology 22, 1315 - 1316.

Green, P. J. (1995). Reversible jump Markov chain Monte Carlo computation and Bayesian model determination. Biometrika 82(4), 711-32.

Hamilton, J. D. (1989, March). A new approach to the economic analysis of nonstationary time series and the business cycle. Econometrica 57(2), 357-384. 
MacDonald, I. L. and W. Zucchini (1997). Monographs on Statistics and Applied Probability 70: Hidden Markov and Other Models for Discrete-valued Time Series. Chapman \& Hall/CRC.

Mackay, R. (2002). Estimating the order of a hidden Markov model. Canadian Journal of Statistics 30(4), $573-589$.

MATLAB (2012). version 7.14 .0 (R2012a). Natick, Massachusetts: The MathWorks Inc.

Nam, C. F. H., J. A. D. Aston, and A. M. Johansen (2012). Quantifying the uncertainty in change points. Journal of Time Series Analysis 33(5), 807-823.

Peng, J.-Y., J. A. D. Aston, and C.-Y. Liou (2011). Modeling time series and sequences using Markov chain embedded finite automata. International Journal of Innovative Computing Information and Control 7, 407-431.

R Development Core Team (2011). R: A Language and Environment for Statistical Computing. Vienna, Austria: R Foundation for Statistical Computing. ISBN 3-900051-07-0.

Rabiner, L. (1989, February). A tutorial on hidden Markov models and selected applications in speech recognition. Proceedings of the IEEE 77(2), 257-286.

Robert, C. P., T. Rydén, and D. M. Titterington (2000). Bayesian inference in hidden Markov models through the reversible jump Markov chain Monte Carlo method. Journal of the Royal Statistical Society: Series B (Statistical Methodology) 62(1), 57-75.

Scott, S. (2002). Bayesian methods for hidden Markov models: Recursive computing in the 21st century. Journal of the American Statistical Association 97(457), 337-351.

Titterington, D. M. (1984). Comments on "Application of the conditional population-mixture model to image segmentation". Pattern Analysis and Machine Intelligence, IEEE Transactions on PAMI-6(5), $656-658$.

Viterbi, A. (1967, April). Error bounds for convolutional codes and an asymptotically optimum decoding algorithm. Information Theory, IEEE Transactions on 13(2), 260 - 269. 